\title{
Application of Intelligent Sensor Technology in Steady State Operation of Power System
}

\author{
Huang Zongjian \\ College of mechanical and electrical engineering, Henan Polytechnic Institute, Nanyang Henan, \\ China
}

\begin{abstract}
Wireless sensor network technology is a new monitoring technology to ensure the stable and safe operation of power system. The network has many advantages: light weight, corrosion resistance, high temperature resistance and so on, which can effectively solve the measurement problems that conventional detection technology can not afford. With its unique physical properties, the sensor technology has a relatively broad application prospect in power system. This paper focuses on the main development achievements of wireless sensor technology, and analyzes its application in power system. This paper focuses on the key technologies in the implementation of WSN. This paper discusses the routing of WSN, low power algorithm of nodes, channel deployment, antenna selection, power supply and network security. At the same time, this paper implements the low-power algorithm of the node. The node can run stably for a long time without outside interference. In this paper, through the research on the key technology of theory, the WSN technology is applied to the field investigation and verification of distribution communication network and transmission line condition monitoring, which can be used as the main technical means of the last kilometer network in the power communication terminal network planning. WSN has a broad application prospect in power system.
\end{abstract}

Keywords: Wireless sensor network, power system, steady state, condition monitoring.

\section{Introduction}

Wireless sensor networks (WSN) develops the traditional sensor information acquisition technology from an independent single mode to the direction of integration, miniaturization, networking and intelligence, and has become an important research hotspot in the field of LT in recent years [1-2]. Wireless sensor network integrates sensor technology, embedded computing technology, distributed information processing technology and communication technology, which can sense and collect the information of monitoring objects in the network distribution area cooperatively and transmit it to the observer. As the carrier and bridge between the objective physical world and the subjective perception world, wireless sensor network provides a different information acquisition and processing technology, which is a revolution of information perception and collection [3].

With its own technical characteristics, wireless sensor network can be widely used in many fields, such as national defense and military, environmental monitoring, urban management, medical and health, $\mathrm{T}$ industry control, antiterrorism and disaster resistance [4-5]. In the field of power system automation, wireless sensor network has been applied to remote meter reading, load forecasting, substation automation, distribution network relay protection, distribution line fault location, transmission line real-time monitoring and early warning. These applications can effectively monitor the operation status of power system, improve the operation efficiency of power system, and make wireless sensor network a useful supplement to the links of power production, transmission, distribution and consumption.

The rise of smart grid has attracted wide attention from government departments, industry and academia. Smart grid integrates the most advanced sensor technology, network technology, communication technology, new energy technology and IT technology into the power system, so that it has the characteristics of self-healing,

ISSN: 0010-8189 
interaction, compatibility, integration, optimization and security that the existing power grid does not have. Not only electrical enterprises such as GE, IBM, ABB and SIEMENS are keen on the research and development of smart grid products, but also information industry leaders such as Intel, Cisco, Google and Microsoft have invested in smart grid business and played a role in the field of grid information processing [6]. Wireless sensor networks can obtain physical information such as power grid operation status and parameters, provide more comprehensive and complete power grid operation data for power grid operation and management personnel, help to make decisions on system control implementation plans and response plans, and will become an effective part of smart grid.

\section{Research trends and characteristics}

\subsection{Research trends}

The early research of wireless sensor network originated from the military project of the United States in the 1970s. In the past 10 years, the rise of wireless sensor network has aroused the extensive attention of domestic and foreign research institutions, which makes the wireless sensor network get a long-term development in the basic theory and supporting technology. At present, wireless sensor network is in the stage from experimental prototype system to large-scale practical application, and its connotation and extension have been constantly enriched and expanded.

In theoretical research, researchers based on wireless sensor network large-scale, self-organizing, multi hop, no partition, no infrastructure. Nodes are isomorphic, low cost, small size, random deployment, limited energy and can not be regenerated, communication and computing capacity are strictly limited [7]. Around the core of low power consumption, we continue to explore topology control, network routing, data fusion, target location, network security and other aspects, so as to extend the network life.

In practical applications, people no longer stick to the traditional sense of wireless sensor network, which emphasizes the harsh conditions such as low power consumption and random dense deployment of nodes, but take the application as the center according to the specific application requirements and conditions. Using mature, simple and feasible technology, all the monitoring systems with the help of wireless communication technology to achieve networking are included in the scope of wireless sensor network research, emphasizing the vitality and great potential of the flexible and convenient wireless communication technology after injecting the network communication function into the traditional independent sensor.

\subsection{Main features}

(1) Application relevance [8]. Under different application background, the hardware platform, software system, network protocol and even architecture of wireless sensor network are not the same. To study the technology of wireless sensor network for each specific application is a significant feature of wireless sensor network design different from traditional network.

(2) Data centric [9]. The basic idea of data centric sensor network is to regard sensor as sensing data stream or sensing data source, wireless sensor network as sensing data space or sensing database, and data management and processing as the application goal of the network. Sensing data management and processing technology is the core technology of data centric sensor networks.

(3) Self organization and self adaptation [10]. Wireless sensor network nodes can be randomly or manually deployed in the monitoring area, without relying on any pre-set network facilities. Nodes have the ability of selforganization and self adaptation, and can automatically configure and manage, coordinate their own behavior through topology control and network protocol, and automatically form an independent network.

(4) Dynamic topology. In addition, the node energy, wireless channel, terrain, weather and other environmental factors make the wireless sensor network have a dynamic and reconfigurable network topology.

ISSN: 0010-8189 
(5) Multi hop network routing. Due to the limited transmit power, the nodes in wireless sensor networks need to forward the intermediate nodes when they communicate with the nodes outside the coverage, which requires the network to have multi hop routing. Moreover, the multi hop routing of wireless sensor networks is completed by the cooperation of ordinary nodes, without the need for special routing equipment.

\section{Application prospect in Smart Grid}

Smart grid covers advanced measurement infrastructure (AMI) and advanced distribution operation (ADO). The close cooperation between advanced transmission operation (ATO) and advanced asset management (AAM) can achieve the goals of safe and stable operation of smart grid, compatibility with distributed generation, improving the utilization of grid assets, improving the efficiency, reliability and power quality of users. Wireless sensor networks are widely used in AMI, ADO, ATO and AAM.

\subsection{Application of WSN in AMI}

AMI is a network processing system used to measure, collect, store, analyze and use users' electricity consumption information. It is mainly composed of smart meter, home area network (Han), local communication network, back haul communication network connected with power company data center, measurement data management system, etc. In general, AMI is the first step of power grid intelligence. AMI connects users and power companies closely, laying the foundation for the realization of smart grid functions such as distribution automation.

Figure 1 shows the AMI system based on WSN. The communication line from WSN gateway to data center can use power line carrier, digital microwave, SDH optical fiber, ASON optical network, GPRS / CDMA satellite communication and other communication media. From WSN gateway to the "last mile" of electric equipment, a complete AMI system is realized by WSN with a series of micro power wireless communication technologies. Micro power wireless communication technology includes Bluetooth, Wi Fi, ZigBee, UWB and so on. Low cost, low power consumption and peer-to-peer communication are three important characteristics and advantages of micro power wireless communication technology. The characteristics of typical micro power wireless communication technology are shown in Table 1.

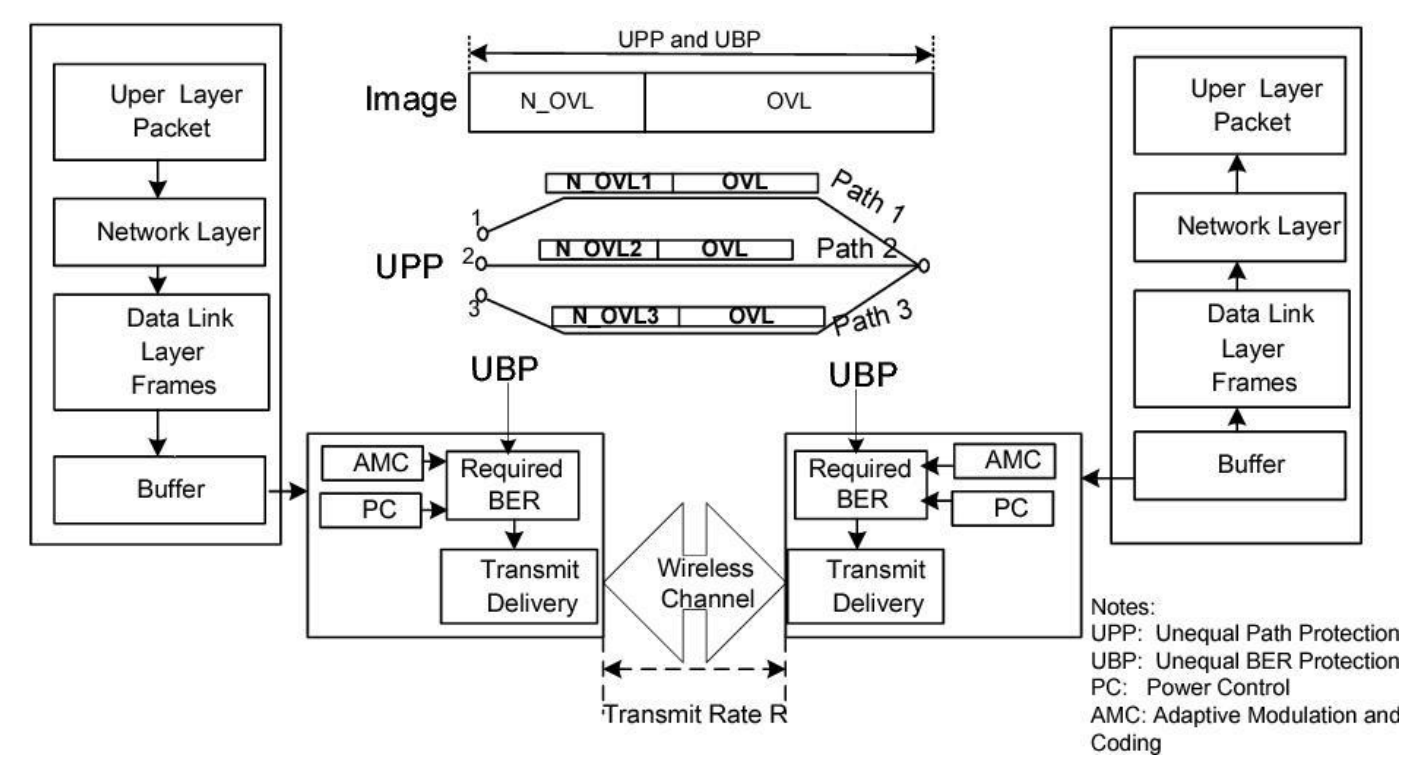

Fig 1: Research of AMI based on WSN

Table 1 Typical micro power wireless communication technology

ISSN: 0010-8189 
CONVERTER MAGAZINE

Volume 2021, No. 1

\begin{tabular}{|c|c|c|c|}
\hline Standard & 802.15 .1 (Bluetooth) & $802.11 \mathrm{~b}$ (WiFi ) & 802.15 .4 (ZigBee) \\
\hline Application area & Substitute peripheral cable & Wireless access & Monitoring and control \\
\hline Bandwidth / $(\mathrm{kb} \cdot \mathrm{a}-1)$ & $1000-3000$ & $11000+$ & $20-250$ \\
\hline Transmission distance & $\begin{array}{c}20 \text { (type 2) } \\
100+\text { (type 1) }\end{array}$ & $100+$ & $100+$ (Amplifier) \\
\hline Number of nodes & 7 & 32000 & 25400 \\
\hline Transmit power / $\mathrm{mA}$ & 45 (type 2) $<150$ (type 1) & $70+$ & 30 \\
\hline Memory overhead / kb & $50+$ & Bandwidth, flexibility & $40+$ \\
\hline Technical advantages & Convenient cost & & \\
\hline
\end{tabular}

WSN technology overcomes the limitations of data point-to-point wireless transmission mode, and uses selforganizing network and automatic relay to realize low-power, long-distance and multi obstacle data transmission. At present, ZigBee technology with low power consumption, low cost and low rate has become a more acceptable standard for Han user interface communication. Through ZigBee technology, the wireless communication between smart meters and home devices is realized, which constitutes the energy management system in the home. Smart home is an application field of WSN, and the emergence of smart grid will eventually achieve this goal. With the development of Li Neng power grid, Han will integrate 3G, 4G network and Internet into people's daily life and become a part of people's modern and convenient life.

\subsection{Application of WSN in ATO}

Transmission network is the backbone of power grid. China will build a strong energy-saving power grid with UHV power grid as the backbone. ATO plays an important role in smart grid. The technical components and functions of ATO mainly include: substation automation, geographic information system (CIS) of power transmission, wide area measurement system (WAMS), high-speed information processing, advanced protection and control, simulation, simulation and visualization tools, advanced transmission network elements, advanced local power grid operation, etc.

Substation automation is inseparable from the support of advanced communication technology. This paper discusses the application of wireless sensor network in substation automation. Among them, a new type of substation automation communication network based on wireless ad hoc sensor network is proposed, the architecture of substation wireless ad hoc sensor network is designed, and the selection of routing protocol is discussed. This paper proposes a wireless sensor network networking scheme based on Clustering Routing for substation automation system with two architectures, and gives a preliminary solution to the key technologies in the implementation of the scheme, such as energy supply of sensor nodes, data fusion and clustering routing algorithm, reliability and security technology, WSN access Ethernet and so on.

Transmission line monitoring system mainly collects the operating environment data of transmission line and transmission tower, including line temperature, humidity, pollution, icing, wind deviation, mountain fire, lightning strike, tower environmental temperature, stress status, etc., and realizes line fault monitoring and management under the condition of multi information integration and fusion, which lays the foundation for digital line. At present, the State Electric Power Construction Research Institute has deployed the wireless sensor network nodes developed by crossbow company on the high-voltage transmission line (see Figure 2), and the sensor network gateway is fixed on the high-voltage transmission tower to monitor the stress, temperature, vibration and other parameters of the long-span transmission line. In addition, the wireless sensor network node with video capture

ISSN: 0010-8189 
function can collect the scene image for disaster warning and realize the whole network visualization.

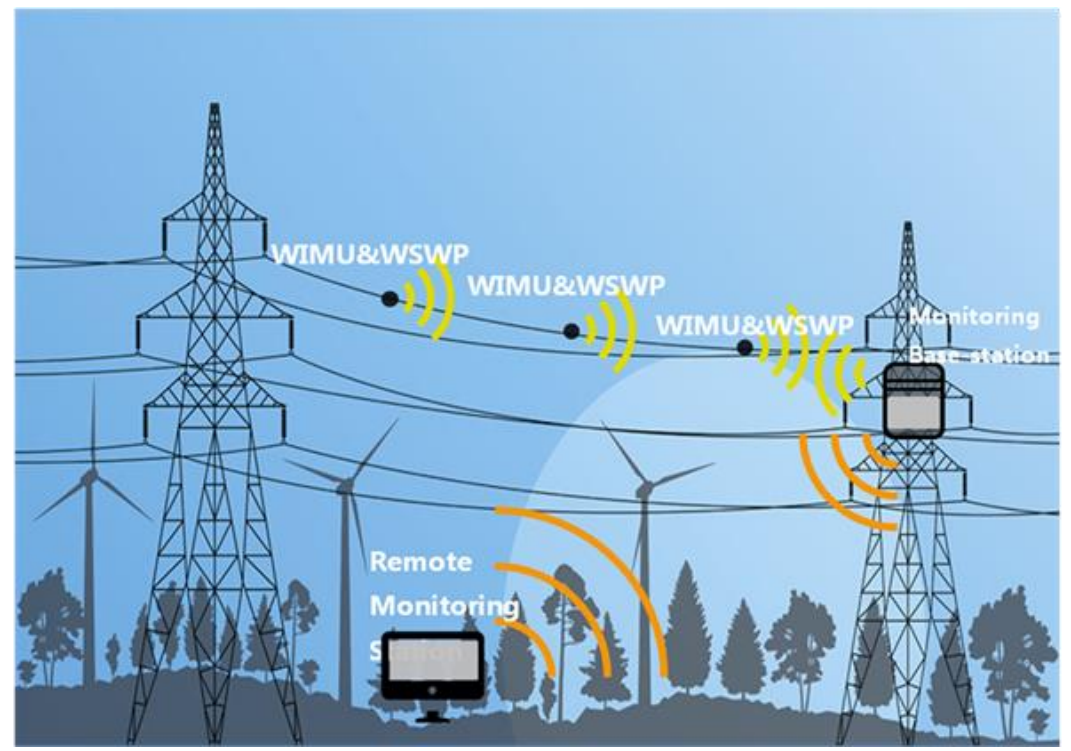

Fig 2: Wireless sensor network nodes on transmission lines

\subsection{Application of WSN in AAM}

AAM uses the information and control of AMI, ADO and ATO, which can improve the utilization rate of equipment, improve the operation efficiency of power grid, reduce line loss and reduce the operation cost of power grid.

In smart grid, AAM can use WSN technology to deploy a large number of sensor nodes in the system, which can provide system parameters and asset status. Through wireless sensor network, the collected real-time information is combined with the operation of optimizing asset use, transmission and distribution network planning, regular maintenance of equipment, engineering design and construction, customer service, work and resource management, simulation and simulation, etc. So as to improve the utilization of assets, optimize the investment of power grid, and reduce the operation cost of operators.

\section{Main technical problems to be solved}

\subsection{Electromagnetic compatibility}

The electromagnetic interference factors of wireless sensor network in power grid mainly include corona interference, radio interference, lightning, radiated electromagnetic field and so on. In the process of data acquisition and transmission, the wireless sensor network applied in smart grid may cause errors due to electromagnetic interference, so the prerequisite for the normal work of wireless sensor network is to be able to withstand the strong electromagnetic interference generated in the case of normal work or fault of power grid. The generation, transmission and reception of electromagnetic interference can not be completely eliminated, but the feasibility design of electromagnetic compatibility can be completed in the case of meeting the requirements of electromagnetic compatibility design objectives. The EMC design of wireless sensor networks should consider not only the normal operation under the electromagnetic environment with specified interference intensity, but also the electromagnetic + interference intensity sent to the outside world within the specified range.

\subsection{Key support technologies}

The key supporting technologies of WSN include node deployment, topology control, routing protocol, clock

ISSN: 0010-8189 
synchronization, energy supply, location technology, data management and fusion, etc.

In smart grid, WSN nodes usually need to be deployed manually, rather than randomly dropped. Wireless sensor networks collect electrical and non electrical quantities in smart grid. In the process of deployment, we should comprehensively consider the number of nodes, sensing range and communication radius, so as to meet the requirements of sensor network connectivity and sensing reliability, and realize the rapid and effective networking of network nodes.

Topology control is one of the core technologies of wireless sensor networks. Through topology control, it can improve the efficiency of routing protocol and MAC protocol, lay the foundation for time synchronization, data fusion, target location and many other aspects, and improve the efficiency and accuracy of data transmission. Smart grid itself has a flexible reconfigurable distribution network topology, and wireless sensor networks need to adapt to it.

Wireless sensor network routing protocol is different according to the specific application. In the smart grid, some applications have higher requirements on the service reliability and real-time performance of communication. Therefore, the routing protocol of wireless sensor network should be designed to meet the QoS requirements according to the stability of the link, the quality of the channel and the topology change. Simulation result is shown in Figure 3.

Time synchronization mechanism is a key mechanism of distributed system infrastructure. Wireless sensor network (WSN) is a new distributed system. The cooperative work of the system requires time synchronization between nodes. In smart grid, it is necessary to consider the synchronization accuracy requirements of specific applications to ensure the time synchronization accuracy of wireless sensor networks.

In the traditional research of wireless sensor networks, it is assumed that the node energy is very limited and non renewable, and all kinds of technologies aim at energy saving. In smart grid, small TA energy collection, breeze power generation, solar cells and other technologies can be used to make wireless sensor network nodes obtain continuous power and ensure the continuous supply of energy, so as to meet the energy demand of large, fast and reliable data transmission in smart grid.

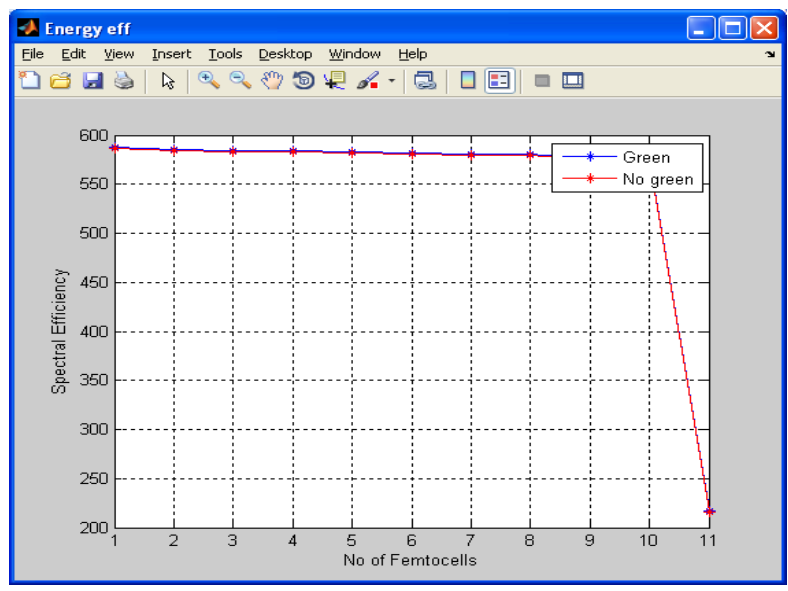

Fig 3: Simulation result

\subsection{Standardized communication}

Smart grid should have open, standard and integrated communication system, which is the basis of smart grid operation. Without comprehensive communication system, there will be no smart grid. Wireless sensor network is not isolated in smart grid, but should be combined with power communication system, including power

ISSN: 0010-8189 
broadband communication (BPL), digital microwave, SDH optical fiber, ASON optical network and other communication technologies. A fully integrated communication system must use common standards. Wireless sensor networks in different applications should follow the corresponding standards to connect people to the power communication network. The standard setting of smart grid is still in the process of further promotion. In the process of promotion, the integration of wireless sensor network and existing communication system should be considered to avoid repeated development.

4.4 Network security technology

Compared with the traditional power communication network, the wireless sensor network applied in smart grid is facing greater security challenges. As the interface between the real physical environment and the information system, wireless sensor network is a deeply embedded network. For different applications, the structure and protocol of wireless sensor network will be different. This feature of wireless sensor network is difficult to apply traditional network security algorithms and protocols to sensor network directly. In addition to considering the confidentiality, integrity and freshness of information acquisition, as well as the recognition of communication entities and message sources, the security issues should be integrated into network routing, clock synchronization, data fusion and other links, so as to ensure the safe, stable and reliable operation of smart grid.

\section{Conclusion}

This paper studies the application of wireless sensor network in smart grid, and analyzes the main technical problems to be solved. From the research status of wireless sensor network in power system, some research results have been achieved, but it has not been tested in large-scale practical application. From the perspective of the development plan of smart grid, the participation of wireless sensor networks is necessary. Therefore, in the context of specific applications, wireless sensor networks that meet the requirements of self-healing, interaction, compatibility, integration, optimization and security of smart grid will be the development trend of its application in smart grid.

\section{References}

[1] Song Lai. Design and Implementation of Mine Electrical Automation Control System Based on Computer and Artificial Intelligence Technology. China Metal Bulletin, 2016 (11): 80-81

[2] Liu Xiangyang, Wang Dan. Design and Research of Electrical Automation Control System Based on Intelligent Technology. Electronic Design Engineering, 2019, V.27; No.414 (16): 72-75

[3] Wen Wenhao. Research on Electrical Automation Control System Based on Artificial Intelligence. Information and Computer (theoretical Edition), 2020, V.32; No.446 (04): 152-153

[4] Liu Xiaoyan. Application of Artificial Intelligence Technology in Electrical Automation Control System. Rural Economy and Technology, 2019, 030 (022): 254-255

[5] Guo Yanan, Wang Yanfei. Application of Artificial Intelligence Technology in Electrical Automation Control. Architectural Engineering Technology and Design, 2018, 16: 4184

[6] Huang Qiang. Application of Artificial Intelligence Technology in Electrical Automation Control. Consumer Guide, 2019, 10: 120

[7] Yin Zhangsheng. Research on Electrical Automatic Control Technology Under Artificial Intelligence Technology. Development Orientation of Building Materials, 2019, 017 (009): 363

[8] Wang Li. Application of Artificial Intelligence in Electrical Control System. Full Text Edition: Engineering Technology, 2016 (6): 175-175

[9] Jiang Fkuan, Zhou Rui. Discussion on the Application of Artificial Intelligence Technology in Electrical Automation Control. Dual Use Technologies and Products, 2018, 6: 96

[10] Yao Linhu. Application of Artificial Intelligence in Electrical Automation Control. Intelligent City Application, 2020, 3 (8): 91

ISSN: 0010-8189 